\title{
Distinct Abnormalities in the Interaction of Purified Types IIA and IIB von Willebrand Factor with the Two Platelet Binding Sites, Glycoprotein Complexes Ib-IX and IIb-IIla
}

\author{
Luigi De Marco,“ Mario Mazzucato,“ Dina De Roia,“ Alessandra Casonato, ${ }^{\star}$ Augusto B. Federici, ${ }^{5}$ \\ Antonio Girolami, ${ }^{*}$ and Zaverio M. Ruggerill \\ ${ }^{*}$ Centro Trasfusionale e Chimica Clinica, Centro di Riferimento Oncologico Aviano, Pordenone, Italy; ${ }^{\ddagger}$ Istituto di Patologia Medica II, \\ Universitá di Padova, Padua, Italy; ${ }^{\S}$ Hemophilia and Thrombosis Center A. Bianchi Bonomi, Universitá di Milano, Milan, Italy; \\ "Roon Research Center for Arteriosclerosis and Thrombosis, Department of Molecular and Experimental Medicine and Committe for \\ Vascular Biology, Scripps Clinic and Research Foundation, La Jolla, California 92037
}

\begin{abstract}
We have studied the interaction of the congenitally abnormal type IIA and IIB von Willebrand factor (vWF) molecules, both lacking the larger multimeric forms, with the two vWF binding sites on platelets, the glycoprotein (GP) Ib-IX and GP IIb-IIIa complexes. Variant as well as normal $(N)$ vWF were purified from plasma. Estimates for binding of subunit molecules per platelet at saturation $\left(B_{\max }\right)$ and dissociation constant in moles/liter $\left(K_{d}\right)$, respectively, were obtained from binding isotherms of ${ }^{125}$ I-labeled vWF, with the following results. In the presence of ristocetin (binding to GP Ib-IX): N, 25,693 and 0.5 $\times 10^{-8}$; IIA, both parameters not measurable; IIB, 17,708 and $0.87 \times 10^{-8}$. After thrombin stimulation (binding to GP IIbIIIa): $\mathrm{N}, 17,059$ and $1.12 \times 10^{-8}$; IIA, 23,751 and $4.87 \times 10^{-8}$; IIB, 19,890 and $2.52 \times 10^{-8}$. Distinct experiments based on measuring the ability of the variant species (from the same patients and one additional IIB patient) to inhibit the binding of normal ${ }^{125} \mathrm{I}-\mathrm{vWF}$ to platelets gave results in agreement with those reported above. Other studies showed that only IIB vWF bound to platelets in the absence of any mediating substance $\left(K_{d}=5.21 \times 10^{-8} \mathrm{~mol} /\right.$ liter and $B_{\max }=9,599$ subunits per platelet) and induced aggregation at a concentration of 10 $\mu \mathrm{g} / \mathrm{ml}\left(3.6 \times 10^{-8} \mathrm{M}\right)$. Thus, IIB vWF binds to GP Ib-IX with high affinity and induces platelet aggregation, whether with or without ristocetin, in spite of the absence of larger multimers. In contrast, the binding of IIA vWF to GP Ib-IX occurs with very decreased affinity, and this defective function may result from specific structural abnormalities rather than just being a reflection of the absence of larger multimeric forms. Both IIA and IIB vWF exhibit decreased affinity for GP IIb-IIIa. In this case, the extent of the defect correlates with the absence of larger multimers. (J. Clin. Invest. 1990. 86:785-792.) Key words: glycoprotein • platelet • von Willebrand disease • von Willebrand factor
\end{abstract}

Parts of this work were presented at the meeting of the American Heart Association, Washington, DC, 14-17 November 1988.

This is Publication No. 6220-MEM from the Research Institute of Scripps Clinic.

Address reprint requests to Dr. Ruggeri, BCR-8, Research Institute of Scripps Clinic, 10666 North Torrey Pines Road, La Jolla, CA 92037.

Received for publication 4 January 1990 and in revised form 6 April 1990.

J. Clin. Invest.

(c) The American Society for Clinical Investigation, Inc.

0021-9738/90/09/0785/08 $\$ 2.00$

Volume 86, September 1990, 785-792

\section{Introduction}

The multimeric structure of von Willebrand factor (vWF) ${ }^{1}$ is considered important for providing high-affinity binding to the platelet membrane $(1,2)$. This occurs by means of functional domains present in each subunit and having recognition specificity for one of two known platelet sites, the glycoprotein (GP) Ib-IX complex and the GP IIb-IIIa complex (3-5). Thus, one large vWF multimer may interact in a multivalent manner with several binding sites on one platelet. The occurrence of bleeding, both in congenital von Willebrand disease and in acquired abnormalities of vWF, is correlated to the absence of larger multimers even in patients with normal total concentration of vWF (1). The two most common congenital variants of von Willebrand disease with abnormalities of multimeric structure are type IIA and type IIB $(6,7)$. The current concepts of pathophysiology suggest that, in the former, vWF fails to polymerize normally or the larger multimers are structurally unstable (8); in the latter, vWF has increased affinity for platelets and, consequently, the larger multimers are removed from the circulation as a result of platelet binding $(7,9-11)$. To date, however, there is no detailed information on the modalities of interaction of IIA and IIB vWF with the corresponding binding sites on platelets.

The association of vWF with both GP Ib-IX and GP IIbIIIa appears to be important in thrombus formation $(12,13)$, and knowledge of the molecular mechanisms responsible for it is likely to provide useful information for understanding the processes involved in normal hemostasis as well as pathological vascular occlusion. In this regard, the study of molecular variants of von Willebrand disease is of particular interest if the existence of specific molecular defects can be correlated to relevant abnormalities of interaction with platelets. To address these issues, we have purified the congenitally abnormal vWF molecule from the plasma of patients with type IIA and IIB von Willebrand disease, and evaluated in a quantitative manner the interaction with GP Ib-IX and GP IIb-IIIa. Our findings demonstrate that IIB vWF can bind to GP Ib-IX in the absence of any mediating or activating molecule, suggesting the existence of a molecular defect resulting in increased affinity for this platelet site. Such increased affinity is also evident in the presence of ristocetin, in spite of the absence of larger multimers. In contrast, IIA vWF interacts with GP Ib-IX with extremely reduced affinity. The severity of this abnormality may reflect the existence of specific structural alterations af-

1. Abbreviations used in this paper: $B_{\max }$, number of molecules bound at saturation; GP, platelet membrane glycoprotein; vWF, von Willebrand factor. 
fecting the GP Ib-binding site in IIA vWF, in addition to the derangement of multimeric structure typical of these patients. Finally, both variant forms of vWF bind to the GP IIb-IIIa complex on activated platelets with decreased affinity. In this case, the extent of the abnormality appears to correlate essentially with the decrease of larger multimers (14).

\section{Methods}

All blood samples were obtained from patients and normal volunteers with their informed consent and in accordance with the declaration of Helsinki. They had taken no medications for at least 2 wk.

Purification of proteins. The purification of vWF was performed as reported $(9,10)$ with the only modification of adding to the starting plasma aprotinin (Sigma Chemical Co., St. Louis, MO) and EDTA, $200 \mathrm{U} / \mathrm{ml}$ and $5 \mathrm{mM}$ final concentrations, respectively. Two different preparations of IIB vWF were obtained from $(a) 1,500 \mathrm{ml}$ of plasma from one patient (D.Z.) previously described in detail $(9,10)$ and $(b)$ $2,000 \mathrm{ml}$ of plasma from patient C.A., who fulfilled the criteria for the diagnosis of IIB von Willebrand disease (7). One preparation of IIA vWF was obtained from $2,300 \mathrm{ml}$ of plasma from patient D.M., who fulfilled the criteria for the diagnosis of IIA von Willebrand disease (6). In addition, normal vWF was purified from $\sim 1$ liter of plasma either obtained from a single volunteer or pooled from four different donors. Purified vWF was contained in the first protein peak (detected by light absorbance at $280 \mathrm{~nm}$ ) eluting at the void volume of a $2.5 \times 80-\mathrm{cm}$ gel filtration column of Sepharose 4B-CL (Pharmacia LKB Biotechnology Inc., Piscataway, NJ). The peaks of IIA and IIB vWF appeared in the same position as that of normal vWF, but their maximal height was smaller because of the lower content of vWF, and the area corresponding to the larger multimers was less represented. The final yields, based on recovery of total ristocetin cofactor activity units as percentage of those present in the starting plasma, were $20-25 \%$ for normals, $3.5 \%$ for patient D.Z., $5.2 \%$ for patient C.A., and $4 \%$ for patient D.M In the latter, the ristocetin cofactor activity in plasma was $0.12 \mathrm{U} / \mathrm{ml}(1$ $\mathrm{U} / \mathrm{ml}$ is the activity of "standard" normal plasma); the specific ristocetin cofactor activity of purified vWF was $14.7 \mathrm{U} / \mathrm{mg}$ of protein (as opposed to $155 \mathrm{U} / \mathrm{mg}$ for normal vWF). The reported ristocetin cofactor activity of IIB vWF is between 48 and $68 \mathrm{U} / \mathrm{mg}(10)$. The final yield of patient $\mathrm{vWF}$ was lower than normal because, in agreement with previous findings $(9,10)$, the decreased concentration of larger multimers determines a lower recovery of vWF in the initial cryoprecipitation step.

Analysis of the purified $\mathrm{VWF}$ on reduced polyacrylamide gels showed a major band with apparent mobility of $220 \mathrm{kD}$ in both normals and patients and some smaller bands, owing to proteolytic degradation, which were slightly increased in patient D.M. (15). All the bands stained with Coomassie Blue were identified as VWF by immunoblotting with specific monoclonal antibodies (16). Purified normal, IIB, and IIA vWF were stored in $0.02 \mathrm{M}$ Tris- $\mathrm{HCl}, 0.15 \mathrm{M} \mathrm{NaCl}$, pH 7.3 (Tris buffer), and kept at $-70^{\circ} \mathrm{C}$. The concentration of vWF was measured by the method of Lowry et al. (17).

Sialic acid measurement. The sialic acid content of vWF was measured according to the method of Warren (18) after incubating purified vWF with $0.1 \mathrm{~N} \mathrm{H}_{2} \mathrm{SO}_{4}$ for $60 \mathrm{~min}$ at $100^{\circ} \mathrm{C}$. Samples were tested in duplicate or triplicate. $N$-acetyl-neuraminic acid was used as a standard.

Radioiodination of $v W F$. This was performed with ${ }^{125} I$ using Iodogen (Pierce Chemical Co., Rockford, IL) according to the method of Fraker and Speck (19). The specific activity of normal, IIA, and IIB vWF was between 0.3 and $0.6 \mathrm{mCi} / \mathrm{mg}$.

Monoclonal antibodies. Monoclonal antibodies against GP Ib and the GP IIb-IIIa complex were prepared and characterized as described $(20,21)$. The antibodies used were $\mathrm{LJ}-\mathrm{Ib} 1$, specific for GP Ib, and LJ-P5 and LJ-CP8 directed against the GP IIb-IIIa complex. LJ-Ib1 inhibits VWF binding to GP Ib; LJ-P5 inhibits vWF but not fibrinogen or fibronectin binding to GP IIb-IIIa; LJ-CP8 inhibits binding of all adhesive proteins that interact with GP IIb-IIIa. Purified IgG and monovalent $\mathrm{Fab}^{\prime}$ were prepared as reported $(22,23)$ and kept in Tris buffer.

Aggregation studies. Citrated platelet-rich plasma from a severe afibrinogenemic patient was prepared as described from blood containing $0.011 \mathrm{~mol} /$ liter of trisodium citrate (24). All aggregation studies were performed with a Chronolog aggregometer (Chronolog Corp., Haverton, PA) in siliconized glass cuvettes at $37^{\circ} \mathrm{C}$ with a constant stirring at $1,200 \mathrm{rpm}$ and a platelet count of $2.5 \times 10^{8} \mathrm{ml}$. Washed platelets were prepared according to a modification of the albumin density gradient technique (25) as reported elsewhere (5).

Binding experiments. Binding of ${ }^{125} \mathrm{I}$-labeled normal, IIA, and IIB (patient C.A.) vWF to washed normal platelets in the presence of ristocetin (binding to GP Ib-IX) or after stimulation with $\alpha$-thrombin (binding to GP IIb-IIla) was measured as previously reported in detail (5). In brief, platelets at the final count of $1.44 \times 10^{8} / \mathrm{ml}$ were incubated with ${ }^{125} \mathrm{I}$-labeled vWF at concentrations between 0.27 and 25 $\mu \mathrm{g} / \mathrm{ml}$, in the presence of ristocetin $(1.2 \mathrm{mg} / \mathrm{ml})$ or after stimulation with $\alpha$-thrombin $(0.8 \mathrm{NIH} \mathrm{U/ml;} \mathrm{a} \mathrm{gift} \mathrm{of} \mathrm{Dr.} \mathrm{J.} \mathrm{W.} \mathrm{Fenton} \mathrm{II,} \mathrm{Griffin}$ Laboratories, New York State Department of Health, Albany, NY). All incubations were carried out in polypropylene tubes, at $22-25^{\circ} \mathrm{C}$ for $30 \mathrm{~min}$, after which separation of platelet-bound radioactivity from free ligand was achieved by centrifugation through $20 \%$ sucrose, as reported (5). Radioactivity was counted in a $\gamma$-scintillation spectrometer (Packard Instrument Co., Laguna Hills, CA), and the amount of vWF bound to platelets was calculated taking into account the specific activity of the radiolabeled molecule. In some experiments, the binding of normal and IIB vWF to thrombin-stimulated platelets was measured in the presence of the anti-GP Ib monoclonal antibody LJ-Ib1. This was done to prevent the direct binding of IIB vWF to GP Ib-IX (see below) and to evaluate whether interaction with this site could affect the parameters of binding to GP IIb-IIIa. Experiments based on inhibition of binding were performed using a fixed amount of ${ }^{125} \mathrm{I}$-labeled normal vWF $(1.2 \mu \mathrm{g} / \mathrm{ml})$ and increasing concentrations of unlabeled normal, IIA or IIB vWF, between 0.33 and $80 \mu \mathrm{g} / \mathrm{ml}$. In this case, unlabeled and labeled ligands were added to platelets in rapid succession one after the other, either in the presence of ristocetin or after stimulation with $\alpha$-thrombin, as described above. The rest of the procedure was performed as described above for the generation of binding isotherms with labeled ligands at varying concentrations. Direct binding of ${ }^{125} \mathrm{I}$-labeled normal or IIB vWF (patient C.A.) was measured using normal platelet-rich plasma in the absence of any inducing molecule, as previously described for asialo vWF (26). The final platelet count was $2.5 \times 10^{8} / \mathrm{ml}$, and the vWF concentration varied between 1.6 and $35 \mu \mathrm{g} / \mathrm{ml}$. The incubation mixtures were kept at room temperature $\left(22-25^{\circ} \mathrm{C}\right)$ or at $37^{\circ} \mathrm{C}$, as specified, without stirring for $30 \mathrm{~min}$, after which separation of platelet bound from free ligand was achieved by centrifugation through $20 \%$ sucrose, as previously described (5), followed by measurement of the bound radioactivity.

The binding parameters were calculated using the computer-assisted program LIGAND (27), assuming a subunit molecular mass of $275 \mathrm{kD}$ for each of the purified vWF preparations. Direct binding isotherms were analyzed using the "Hot" option of the program, whereas inhibition curves were analyzed using the "Drug" option. Nonspecific (nonsaturable) binding was calculated as a fitted parameter. The values obtained in this manner were in close agreement with those measured in the presence of monoclonal antibodies that inhibit binding to either GP Ib-IX or GP IIb-IIIa. The antibodies used were $\mathrm{LJ}-\mathrm{Ib} /$ and $\mathrm{LJ}-\mathrm{CP} 8$, respectively, used at a concentration $(120 \mu \mathrm{g} / \mathrm{ml})$ in excess of that necessary to obtain saturation of the corresponding epitopes and maximal inhibitory activity $(20,28)$. It was not possible to measure nonsaturable binding in the presence of excess amount of unlabeled ligands because of the limited quantities of purified patient vWF available.

\section{Results}

Characterization of purified IIA and IIB $v W F$. The distribution of multimeric forms in purified IIA and IIB vWF was 
comparable to that seen in the plasma of the same patients before purification. In particular, the decrease in multimers of intermediate size was more pronounced in IIA than IIB vWF, but both preparations lacked the larger multimers of normal plasma (Fig. 1). Purified IIB vWF from patient D.Z. induced platelet aggregation when added to citrated platelet-rich plasma at concentrations as low as $5-7 \mu \mathrm{g} / \mathrm{ml}$, whereas vWF from patient C.A. induced aggregation at $10 \mu \mathrm{g} / \mathrm{ml}$. No aggregation was seen with IIA or normal vWF at concentrations up to $300 \mu \mathrm{g} / \mathrm{ml}$. The sialic acid content of purified IIA and IIB vWF was within normal values (Table I).

Binding studies. The interaction of purified vWF with the platelet GP Ib-IX and GP IIb-IIIa binding sites was evaluated either in the presence of ristocetin or after platelet activation with $\alpha$-thrombin, respectively. In a first set of experiments, binding was measured using ${ }^{125} \mathrm{I}$-labeled purified vWF. The dose-dependent association of normal, IIA, and IIB vWF (from patient C.A.) with GP Ib-IX is shown in Fig. 2. Scatchard-type analysis of the binding isotherms using the computer-assisted program LIGAND (27) demonstrated that normal and IIB vWF bound in a saturable manner, whereas no regression could be obtained for IIA vWF (Fig. 2). Nevertheless, an anti-GP Ib monoclonal antibody could inhibit about two-thirds of the total binding measured at each concentration of IIA vWF tested, suggesting that a specific interaction occurred, although the affinity was too low to be calculated from the data points available. The limiting factor for testing higher concentrations was, in this case, the amount of purified IIA vWF that could be obtained from the patient. The values of $B_{\max }$ and $K_{d}$ calculated for the ristocetin-dependent vWF binding to GP Ib are reported in Table II. No estimates could be obtained for IIA vWF, whereas IIB vWF exhibited binding parameters not dissimilar from normal. In particular, the $K_{\mathrm{d}}$

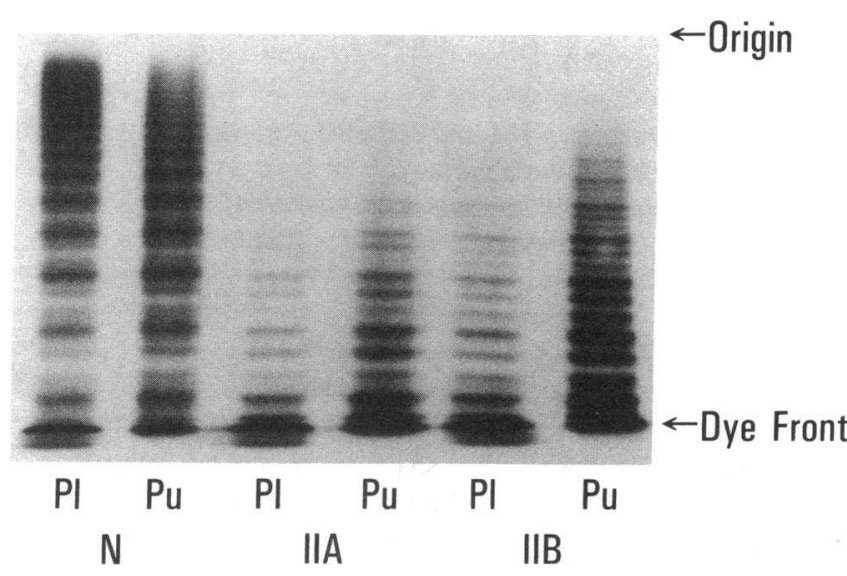

Figure 1. Multimeric composition of purified vWF preparations. Each lane contains $20 \mu \mathrm{l}$ of citrated platelet-poor plasma $(P I)$ or 5-10 ng of purified vWF $(P u)$ from either a normal control $(N)$ or patients with type IIA (patient D.M.) or type IIB (patient C.A.) von Willebrand disease. Electrophoresis was performed in an agarose gel containing sodium dodecyl sulfate, and VWF multimers were detected by autoradiography after reaction with a radiolabeled specific anti-vWF antibody (29). The arrow at the top indicates the interface between stacking and running gel, the one at the bottom indicates the dye front. Cathode was at the top. Note the abnormal multimeric structure in the samples from the patients, with absence or marked decrease of large and intermediate multimers both in plasma and in the purified preparations.
Table I. Sialic Acid Content of Purified IIA and IIB vWF

\begin{tabular}{lc}
\hline \multicolumn{1}{c}{ Source } & Sialic acid \\
\hline & $n m o l / m g$ of $v W F$ \\
Patient D.M. (IIA vWF) & 159 \\
Patient D.Z. (IIB vWF) & 170 \\
Patient C.A. (IIB vWF) & 160 \\
Normal $(n=4)$ & $158 \pm 17$ \\
\hline
\end{tabular}

Results in normal represent the mean \pm 1 SD of four different preparations.

values were approximately the same, in spite of the absence of larger multimers in IIB vWF. As discussed below, this means that the affinity of IIB vWF for GP Ib-IX is greater than normal.

The binding isotherms obtained for the thrombin-induced binding of vWF to GP IIb-IIIa are shown in Fig. 3. In this case, Scatchard-type analysis demonstrated saturable binding for all three types of vWF tested. As summarized in Table II, both IIA and IIB vWF exhibited similar $B_{\max }$ but higher $K_{\mathrm{d}}$ values than normal vWF. The affinity of interaction was lower for IIA than IIB vWF, in accordance with the more pronounced defect in multimeric structure.

A different experimental approach was used to confirm the characteristics of the interaction of IIA and IIB vWF with

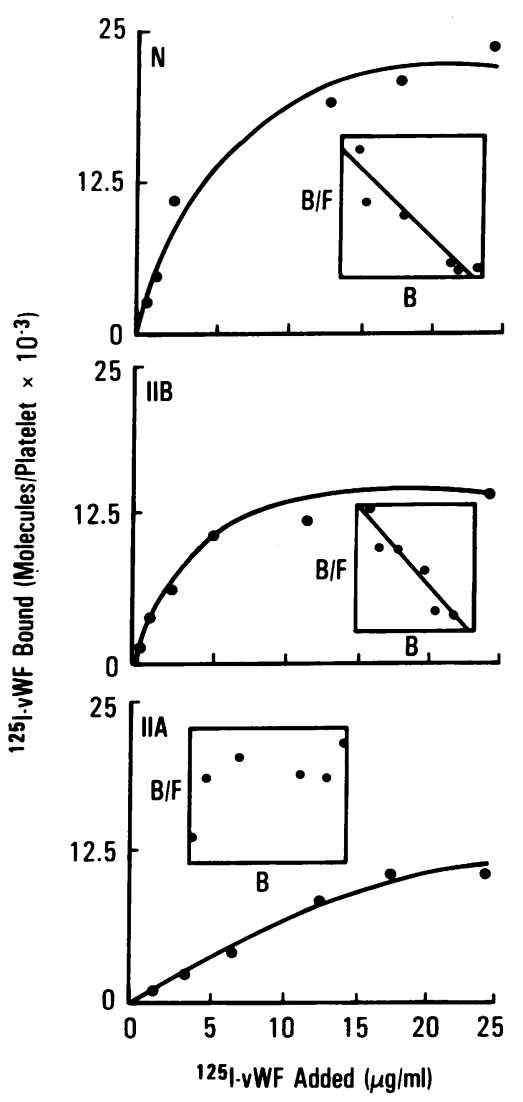

Figure 2. Binding isotherms for vWF interaction with GP Ib-IX in the presence of ristocetin. Purified, ${ }^{125}$ I-labeled vWF, either from a normal individual (top), a IIB von Willebrand disease patient (C.A.; middle), or a IIA von Willebrand disease patient (D.M.; bottom), was incubated at the indicated concentrations with washed normal platelets $\left(1.44 \times 10^{8}\right.$ / $\mathrm{ml}$ ) in the presence of $1.2 \mathrm{mg} / \mathrm{ml}$ of ristocetin. After $30 \mathrm{~min}$ of incubation at $22-25^{\circ} \mathrm{C}$, the amount of bound vWF was determined as described in Methods. The points reported in these graphs represent specific binding, after subtraction of the nonsaturable binding calculated as a fitted parameter from total binding (27). The insets show the computer-generated Scatchard-type analysis of the experimental data (27). Note the lack of linear regression for IIA vWF. 
Table II. Parameters of Radiolabeled IIA and IIB vWF Interaction with GP Ib-IX (Ristocetin-mediated) and GP IIb-IIIa (Thrombin-induced) Calculated from Binding Isotherms

\begin{tabular}{lcclccc}
\hline & \multicolumn{2}{c}{ Ristocetin $(1.2 \mathrm{mg} / \mathrm{ml})$} & & \multicolumn{2}{c}{ Thrombin $(0.8 \mathrm{U} / \mathrm{ml})$} \\
\cline { 2 - 3 } \cline { 5 - 6 } & \multicolumn{2}{c}{$K_{\mathrm{d}}$} & $B_{\max }$ & & $K_{\mathrm{d}}$ & $B_{\max }$ \\
\hline & $\times 10^{-8} M$ & & & $\times 10^{-8} M$ & \\
IIA vWF (D.M.) & $\mathrm{NM}$ & $\mathrm{NM}$ & & $4.87 \pm 1.02$ & $23,751 \pm 4,368$ \\
IIB vWF (C.A.) & $0.87 \pm 0.12$ & $17,708 \pm 5,000$ & & $2.52 \pm 0.25$ & $19,890 \pm 1,449$ \\
Normal ( $n=3)$ & $0.5 \pm 0.18$ & $25,963 \pm 4,323$ & & $1.12 \pm 0.30$ & $17,059 \pm 2,408$
\end{tabular}

Washed platelets (final platelet count $1.44 \times 10^{8} / \mathrm{ml}$ ) were incubated for 30 $\mathrm{min}$ at room temperature with increasing concentrations of radiolabeled normal or variant $\mathrm{vWF}(0.27-25 \mu \mathrm{g} / \mathrm{ml}$ final concentration) either in the presence of ristocetin or after stimulation with $\alpha$-thrombin. Binding parameters were calculated using the computer-assisted program LIGAND (27). The values reported for the patients represent the estimated parameter \pm the SE of the estimate (27). The values reported for the normal controls are the mean of three independent estimates \pm 1 SD. Patient samples were tested twice and the results obtained were in good agreement. Only the results from one experiment are reported here. NM, not measurable, i.e., no defined value could be obtained by analysis of the binding isotherm.

platelets. In this second set of experiments, the binding of a constant amount of normal ${ }^{125} \mathrm{I}$-labeled vWF, corresponding approximately to its $K_{\mathrm{d}}(1.2 \mu \mathrm{g} / \mathrm{ml})$, was measured in the presence of varying amounts of unlabeled normal, IIA, or IIB vWF. By using the computer-assisted program LIGAND (27), estimates for the $B_{\max }$ and $K_{\mathrm{d}}$ values of the competing unla-

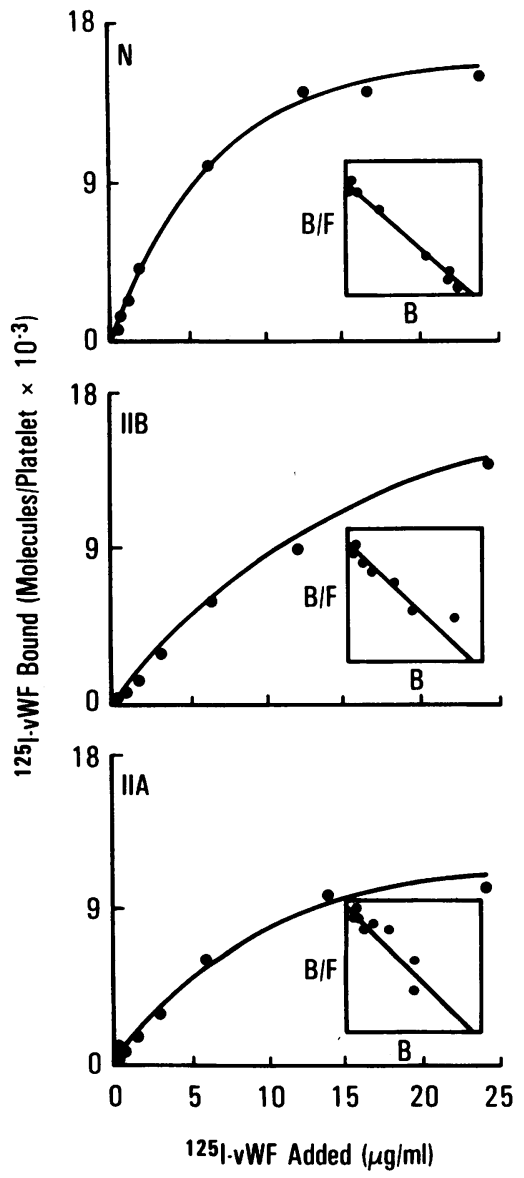

Figure 3. Binding isotherms for VWF interaction with GP IIb-IIIa after thrombin stimulation of platelets. The same samples and experimental conditions described in the legend to Fig. 2 were used for these experiments, except that, instead of adding ristocetin, platelets were stimulated with $0.8 \mathrm{NIH} \mathrm{U} / \mathrm{ml}$ of $\alpha$-thrombin before the addition of labeled ligands. Evaluation of the experimental data was as described in the legend to Fig. 2. Note that, in this case, a linear regression was obtained by Scatchard-type analysis (shown in the insets) for all three types of vWF tested. beled species were derived from the dose-dependent inhibition curves, assuming that labeled and unlabeled species bound to the same sites, and using as estimated $K_{\mathrm{d}}$ of the labeled ligand the value derived from the binding isotherms of normal vWF shown above.

As shown in Table III, unlabeled normal and IIB vWF inhibited the binding of normal ${ }^{125} \mathrm{I}-\mathrm{vWF}$ to GP Ib-IX, and the estimated binding parameters were in good agreement with those obtained from binding of the corresponding radiolabeled species (Table II). It should be noted that two different samples of purified IIB vWF were tested in these experiments, and the results were similar. In particular, both samples of IIB vWF showed a lower $K_{d}$ value (greater affinity) than normal vWF, in spite of the absence of larger multimers in the IIB vWF preparations. These results are different from those observed with normal vWF preparations lacking the larger multimers. As reported by Federici et al. (14) in a separate study performed with the same experimental approach, the $K_{d}$ value for binding to GP Ib-IX in the presence of ristocetin was $0.46 \times 10^{-8} \mathrm{M}$ for fractions of normal vWF containing larger multimers, as opposed to $6.17 \times 10^{-8} \mathrm{M}$ for fractions containing smaller multimers. Thus, the affinity of IIB vWF for GP Ib-IX is much greater than that of normal multimers of equivalent size. Opposite findings were obtained with IIA vWF. In accordance with the results of direct binding experiments, it was not possible to estimate binding parameters for the interaction of unlabeled IIA vWF with GP Ib-IX in the presence of ristocetin, since there was only minimal inhibition of normal vWF binding with this purified molecule. Even when used at a 70-fold excess concentration over radiolabeled normal vWF, IIA vWF inhibited $<50 \%$ of binding. Instead, inhibition of normal vWF binding to GP IIb-IIIa was effectively achieved with all the samples tested. The parameters estimated for IIA and IIB vWF (Table III) were in good agreement with those obtained from isotherms of the corresponding radiolabeled species

Table III. Parameters of IIA and IIB vWF Interaction with GP Ib-IX (Ristocetin-mediated) and GP IIb-IIIa (Thrombin-induced) Calculated from Inhibition of Normal $v W F$ Binding

\begin{tabular}{|c|c|c|c|c|}
\hline & \multicolumn{2}{|c|}{ Ristocetin $(1.2 \mathrm{mg} / \mathrm{ml})$} & \multicolumn{2}{|c|}{ Thrombin $(0.8 \mathrm{U} / \mathrm{ml})$} \\
\hline & $K_{d}$ & $B_{\max }$ & $K_{\mathrm{d}}$ & $B_{\max }$ \\
\hline & $\times 10^{-8} \mathrm{M}$ & & $\times 10^{-8} \mathrm{M}$ & \\
\hline IIA vWF (D.M.) & NM & NM & $8.90 \pm 2.24$ & $16,900 \pm 1,276$ \\
\hline IIB vWF (C.A.) & $0.55 \pm 0.06$ & $18,747 \pm 1,674$ & $2.41 \pm 0.63$ & $22,574 \pm 4,831$ \\
\hline IIB vWF (D.Z.) & $0.28 \pm 0.08$ & $19,675 \pm 5,025$ & $3.01 \pm 0.09$ & $15,678 \pm 1,483$ \\
\hline Normal $(n=3)$ & $0.78 \pm 0.19$ & $15,384 \pm 2,474$ & $1.37 \pm 0.40$ & $18,553 \pm 1,933$ \\
\hline
\end{tabular}

Washed platelets (final platelet count $1.44 \times 10^{8} / \mathrm{ml}$ ) were incubated with a fixed concentration of radiolabeled normal vWF $(1.2 \mu \mathrm{g} / \mathrm{ml}$ final concentration) and increasing concentrations of either unlabeled normal vWF or IIA vWF (patient D.M.) or IIB vWF (patients C.A. and D.Z.). The final concentration of unlabeled vWF was between 0.33 and $80 \mu \mathrm{g} / \mathrm{ml}$. The $K_{d}$ and $B_{\max }$ values for the competing unlabeled species were calculated using the "Drug" option of the computer-assisted program LIGAND (27). The values reported for the patients represent the estimated parameter \pm the standard error of the estimate (27). The values reported for the normal controls are the mean of three independent estimates \pm 1 SD. Samples from patients D.M. and C.A. were tested twice, but only the results of one experiment are reported here. Both experiments gave values in good agreement. NM, not measurable, i.e., no defined value could be obtained by analysis of the inhibition curves. 
(Table II), and both variant molecules exhibited lower affinity than normal vWF for binding to GP IIb-IIIa. Again, the defect was more pronounced for IIA vWF. In this case, the results are in good agreement with those obtained by Federici et al. (14) using fractions of normal vWF enriched in either larger or smaller multimers, suggesting a similar behavior for normal as well as IIA and IIB vWF multimers of equivalent size.

Only IIB vWF exhibited a measurable interaction with unstimulated platelets in the absence of any inducing substance. In citrated platelet-rich plasma, binding occurred in a saturable manner, with an estimated $K_{d}$ value of $5.21 \times 10^{-8} \mathrm{M}$ and estimated $B_{\max }$ of 9,599 vWF subunit molecules bound per platelet (Fig. 4). This interaction was abolished by the anti-GP Ib monoclonal antibody, LJ-Ib1, known to block the binding of vWF to platelets in the presence of ristocetin, thus demonstrating that the site involved in binding IIB vWF to unstimulated platelets is primarily the GP Ib-IX complex.

The ability of IIB vWF to bind directly to GP Ib-IX had a minimal effect on the interaction of the molecule with thrombin-stimulated platelets. This was tested by measuring binding of ${ }^{125} \mathrm{I}$-labeled IIB vWF to activated platelets in the presence or absence of the anti-GP Ib antibody LJ-Ib1, which abolishes the binding function of this site. Whereas $B_{\max }$ was basically unchanged (19,890 without, and 21,915 with antibody), the $K_{d}$ showed a modest increase $\left(2.52 \times 10^{-8} \mathrm{M}\right.$ without, and 3.68 $\times 10^{-8} \mathrm{M}$ with antibody), suggesting that binding to GP $\mathrm{Ib}$ may lead to a slight overestimation of the affinity of IIB vWF for GP IIb-IIIa. The anti-GP Ib antibody had no effect on the binding of normal vWF to thrombin-stimulated platelets.

Aggregation studies. The purpose of these experiments was to evaluate whether purified IIA vWF, which had the lowest affinity for GP IIb-IIIa of all the species tested, could support platelet aggregation induced by ADP. This was tested in afibrinogenemic citrated platelet-rich plasma, in which aggregation in response to ADP is observed when supra-normal concentrations of purified vWF are added (24). Aggregation occurred with IIA vWF, but to a lesser extent than with equivalent concentrations of normal vWF (Fig. 5). In either case, aggregation was inhibited by the monoclonal antibody LJ-P5, which is known to selectively block vWF binding GP IIb-IIIa (21), demonstrating that this interaction is necessary for aggregation under the conditions tested (Fig. 5). The lesser efficacy of purified IIA vWF in supporting aggregation under these conditions is consistent with its lower affinity for the GP IIb-IIIa binding site involved in the response.

\section{Discussion}

The results of the present studies demonstrate in a quantitative manner that the congenitally defective type IIA and IIB vWF molecules exhibit distinct abnormalities in their interaction with the platelet vWF binding sites, GP Ib-IX and GP IIb-IIIa complexes. Analysis of vWF binding to platelets must take into account the heterogeneity of molecular forms typical of this protein. Previous experiments have demonstrated a correlation between average molecular mass of normal vWF multimers and affinity for binding to both GP Ib-IX and GP IIbIIIa $(2,14)$. This is most likely explained by the occurrence of multivalent interactions (30) that may confer greater energy to the binding of larger molecules to multiple copies of a given binding site on the platelet membrane. It should be noted that the best fit obtained by Scatchard-type analysis of the present binding isotherms was represented by a straight line, in agreement with another study published previously (31). In a multivalent system, this behavior can be explained by self-association of the bound oligomers on the platelet surface (30). Variations in this regard, possibly owing to differences in the ligand and platelets used, may explain why others (2) have observed the curvilinear Scatchard plots predicted in a simple multivalent system.

With these considerations in mind, the interaction of variant IIA and IIB vWF with GP IIb-IIIa appears to occur according to the predictable behavior for smaller multimers of normal vWF (14). Thus, the greater decrease in affinity observed for IIA vWF is explained by the lack of large and intermediate multimers, as opposed to lack of only the larger ones in IIB vWF. The most logical interpretation of these findings is that both IIA and IIB vWF molecules contain a normal GP IIb-IIIa binding site, and the defective interaction is only a consequence of the abnormal multimeric structure. As demonstrated previously (24), vWF binding to GP IIb-IIIa can support agonist-induced platelet aggregation, even though

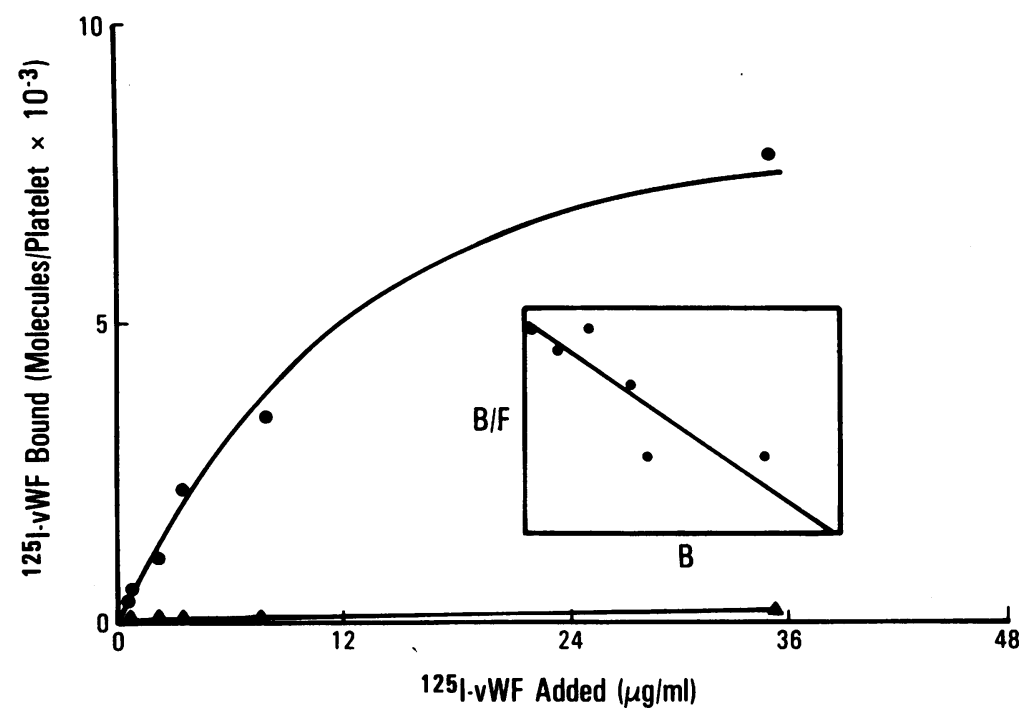

Figure 4. Binding of vWF to unstimulated platelets. Purified, ${ }^{125}$ I-labeled normal vWF ( $\Delta$ ) or IIB vWF ( $\bullet$ ) was added to citrated platelet-rich plasma to give a final platelet count of $2.5 \times 10^{8}$ platelets $/ \mathrm{ml}$ and $\mathrm{vWF}$ concentrations ranging from 1.6 to $35 \mu \mathrm{g} / \mathrm{ml}$. After incubation at room temperature $\left(22-25^{\circ} \mathrm{C}\right)$ for 30 min with no stirring, binding was measured after separating the platelets from unbound protein by centrifugation through $20 \%$ sucrose. The inset represents a Scatchard-type plot of the binding data, generated with the computer-assisted program LIGAND (27). For the purpose of calculation, vWF was assumed to have a molecular mass of $275 \mathrm{kD}$, corresponding to the estimated size of the mature subunit. Note the lack of binding of normal vWF. 


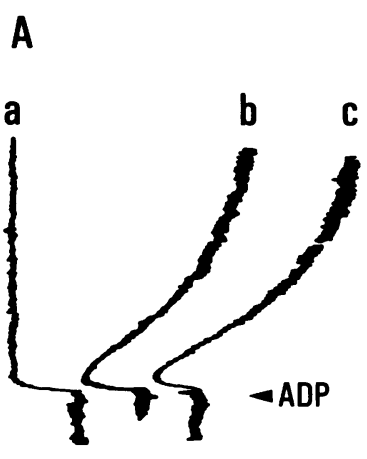

B

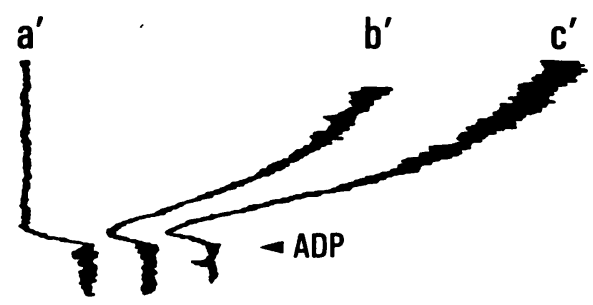

INCREASED LIGHT TRANSMITTANCE

Figure 5. Platelet aggregation mediated by normal vWF and IIA vWF. Citrated platelet-rich plasma was obtained from a patient with congenital afibrinogenemia (24) and used at final platelet count of $2.5 \times 10^{8} / \mathrm{ml}$. (A) Purified IIA vWF or $(B)$ normal vWF was added at final concentrations of $38 \mu \mathrm{g} / \mathrm{ml}$ (traces $b$ and $b^{\prime}$ ) or $76 \mu \mathrm{g} / \mathrm{ml}$ (traces $c$ and $c^{\prime}$ ). Control curves (traces $a$ and $a^{\prime}$ ) contained $76 \mu \mathrm{g} / \mathrm{ml}$ of $\mathrm{vWF}$ and $200 \mu \mathrm{g} / \mathrm{ml}$ of Fab' of monoclonal antibody LJ-P5 (21). Aggregation was induced by the addition, at arrow, of ADP at the final concentration of $2.5 \mu \mathrm{M}$. Note the decreased initial rate and extent of aggregation, as well as the decreased light scattering, observed with IIA vWF as compared to normal vWF. Note also that aggregation is completely abolished by the anti-GP IIb-IIIa monoclonal antibody LJ-P5, which has been shown to inhibit vWF but not fibrinogen interaction with this platelet site (21). Thus, the aggregation observed is mediated by vWF binding to GP IIb-IIIa (24).

with traditional aggregometry this can only be shown in the absence of fibrinogen. In accordance with the results obtained with smaller multimers of normal vWF (14), the decreased binding affinity of IIA vWF for GP IIb-IIIa is well reflected in the decreased ability to support platelet aggregation. Not only were the initial rate and overall extent of aggregation decreased, but the size of aggregates was also smaller with IIA vWF than with normal vWF, as judged by the decreased light scattering observed in the aggregometer (see Fig. 5) and by visual inspection.

In view of the fact that $\mathrm{VWF}$ interaction with GP IIb-IIIa is likely to play a functional role in the formation of platelet thrombi, particularly under flow conditions characterized by high wall shear stress $(13,32)$, the demonstrated defective interaction of IIA vWF with GP IIb-IIIa may play a pathophysiological role in the bleeding diathesis of these patients. The same is presumably true for IIB von Willebrand disease, if one assumes that the interaction of VWF with each of its two platelet binding sites has specific functional significance, and the direct binding of IIB vWF to unstimulated platelets is not likely to counter its decreased affinity for GP IIb-IIIa. In fact, the affinity of IIB vWF for binding to thrombin-stimulated platelets is lower than normal in spite of the possible concurrent interaction with GP Ib-IX.

At variance with the results observed for binding to GP IIb-IIIa, the contrasting defects in the interaction of IIA and IIB vWF with GP Ib-IX are likely to be the consequence of structural abnormalities that transcend the derangement in multimeric structure. The present studies provide the first direct estimate of the dissociation constant for the binding of IIB vWF to GP Ib-IX in unstimulated citrated platelet-rich plasma, a situation under which there is no measurable interaction of intact normal vWF. The value of $14.3 \mu \mathrm{g} / \mathrm{ml}(5.2$ $\left.\times 10^{-8} \mathrm{M}\right)$ is similar to that obtained with desialylated normal vWF under similar experimental conditions (26), even though the total sialic acid content in this preparation of purified IIB vWF, as well as in others analyzed in previous studies (10), was not decreased. In view of the fact that $>60-70 \%$ removal of sialic acid is necessary in order to see a significant aggregating effect with normal vWF (33), it is apparent that a more specific defect must be responsible for causing IIB vWF binding to GP Ib-IX in the absence of inducing substances. The greater than normal affinity of IIB vWF for GP Ib-IX was evident also in ristocetin-dependent binding, in spite of the lack of larger multimers. A comparison of the $K_{\mathrm{d}}$ values reported here with those obtained for normal multimers of corresponding size, reported in a separate publication (14), reveals that the affinity of IIB vWF for GP Ib-IX is $~ 10$-fold greater than normal in the presence of ristocetin. Although the cause, or causes, of this increased affinity remain unknown, at present, unpublished preliminary studies in our laboratory have demonstrated the existence of amino acid substitutions in the GP Ib-binding domain of IIB vWF, and distinct abnormalities are being found in different patients (personal unpublished observation). Indeed, more work is needed before a correlation between these subtle structural abnormalities and the enhanced affinity of IIB vWF for GP Ib-IX can be established.

A good agreement was found between the $K_{d}$ value estimated from binding isotherms and the threshold concentration of IIB vWF necessary to induce platelet aggregation, demonstrating that occupancy of less than half of the total number of binding sites is sufficient to trigger a platelet response. In fact, the two samples from unrelated patients studied here demonstrated a twofold difference in the $K_{d}$ for ristocetin-mediated binding to GP Ib-IX, and this correlated with the threshold concentration for induction of aggregation ( $\mathrm{vWF}$ from patient D.Z. was more active and had lower $K_{d}$ than that from patient C.A.). Barring effects of the purification procedure, which was conducted with identical methodology, these findings may be the consequence of subtle differences in multimeric composition or, possibly, be related to the existence of heterogeneous vWF defects in unrelated IIB patients resulting in abnormal molecules with different affinity for GP Ib-IX (see above).

In contrast to the greater than normal affinity of IIB vWF for GP Ib-IX, the affinity of IIA vWF was so markedly decreased that estimation of a $K_{d}$ value was not possible, whether by direct binding experiments or inhibition of binding studies. Some interaction between IIA vWF and GP Ib-IX occurred, however, as demonstrated by the measurable, albeit markedly decreased, level of ristocetin cofactor activity seen in the plasma of the patient who volunteered for these studies. Nev- 
ertheless, the defect appears to be too extreme to be justified by the abnormality in multimeric structure. In fact, it has been shown that smaller multimers of normal vWF exhibit a concordant decrease in affinity for both GP Ib-IX and GP IIb-IIIa (14). Since the decreased affinity of IIA vWF for binding to GP IIb-IIIa is in good agreement with that of smaller normal multimers, the markedly decreased interaction with GP Ib-IX suggests that, in addition to the absence of larger multimers, a specific alteration, or alterations, in a relevant functional domain may be involved in determining the abnormal IIA vWF binding to GP Ib-IX. Otherwise, one would expect to obtain a $K_{d}$ value similar to that of smaller multimers of normal vWF with equivalent affinity for GP IIb-IIIa, in the order of $5 \times 10^{-8}$ $M$ (14). In contrast, there is a discrepancy in the minimal multimer size required for IIA vWF binding to GP Ib-IX as compared to GP IIb-IIIa, to the extent that multimeric forms that can bind to GP IIb-IIIa cannot bind to GP Ib-IX, or bind with much lower affinity. The molecular abnormality responsible for this altered function does not necessarily involve the sialic acid content of IIA vWF, as this was found to be normal in the sample utilized for the present experiments. It should be kept in mind, however, that a definitive answer to the question of whether abnormalities in multimeric distribution, or specific structural alterations, or a combination of the two, are responsible for the decreased affinity of IIA vWF for GP Ib-IX would require the isolation and functional characterization of homogeneous preparations of multimers of defined size from both normal and IIA vWF. This is not feasible with the currently available methodology, and the interpretation of the results presented here remains open to speculation.

In conclusion, these studies provide evidence that the lack of larger multimers in IIA and IIB vWF is responsible for their decreased affinity for the platelet GP IIb-IIIa binding site. In contrast, the distinct abnormalities in the interaction with GP Ib-IX may involve specific molecular defects that, in the case of IIB vWF, determine increased affinity, while in the case of IIA vWF cause a decrease in affinity greater than predictable on the basis of the alteration in multimeric structure. The nature of these molecular defects remains to be elucidated.

\section{Acknowledgments}

This study was supported by a grant of the Associazione Italiana per la Ricerca sul Cancro (A.I.R.C.); by NATO grant 535/87; by grant P.F. Biotecnologie e Biostrumentazione from Consiglio Nazionale delle Ricerche; and by grants HL-31950 and HL-15491 from the National Institutes of Health.

\section{References}

1. Ruggeri, Z. M., and T. S. Zimmerman. 1987. von Willebrand factor and von Willebrand disease. Blood. 70:895-904.

2. Gralnick, H. R., S. B. Williams, and D. K. Morisato. 1981. Effect of multimeric structure of the factor VIII/von Willebrand factor protein on binding to platelets. Blood. 58:387-397.

3. Fujimura, Y. K. Titani, L. Z. Holland, S. R. Russell, J. R. Roberts, J. H. Elder, Z. M. Ruggeri, and T. S. Zimmerman. 1986. von Willebrand factor: a reduced and alkylated $52 / 48 \mathrm{kDa}$ fragment beginning at amino acid residue 449 contains the domain interacting with platelet glycoprotein Ib. J. Biol. Chem. 261:381-385.

4. Berliner, S., K. Niiya, J. R. Roberts, R. A. Houghten, and Z. M. Ruggeri. 1988. Generation and characterization of peptide-specific antibodies that inhibit von Willebrand factor binding to GP IIb-IIla without interacting with other adhesive molecules: selectivity is conferred by Pro 1743 and other amino acid residues adjacent to the sequence Arg1744-Gly1745-Asp1746. J. Biol. Chem. 263:7500-7505.

5. Ruggeri, Z. M., L. De Marco, L. Gatti, R. Bader, and R. R. Montgomery. 1983. Platelets have more than one binding site for von Willebrand factor. J. Clin. Invest. 72:1-12.

6. Ruggeri, Z. M., and T. S. Zimmerman. 1980. Variant von Willebrand's disease: characterization of two subtypes by analysis of multimeric composition of factor VIII/von Willebrand factor in plasma and platelets. J. Clin. Invest. 65:1318-1325.

7. Ruggeri, Z. M., F. I. Pareti, P. M. Mannucci, N. Ciavarella, and T. S. Zimmerman. 1980. Heightened interaction between platelets and Factor VIII/von Willebrand factor in a new subtype of von Willebrand's disease. N. Engl. J. Med. 302:1047-1051.

8. Ginsburg, D., B. A. Konkle, J. Cox Gill, R. R. Montgomery, P. L. Bockenstedt, T. A. Johnson, and A. Y. Yang. 1989. Molecular basis of human von Willebrand disease: Analysis of platelet von Willebrand factor mRNA. Proc. Natl. Acad. Sci. USA. 86:3723-3727.

9. De Marco, L., A. Girolami, T. S. Zimmerman, and Z. M. Ruggeri. 1985. Interaction of purified IIB von Willebrand factor with the platelet membrane glycoprotein Ib induces fibrinogen binding to the glycoprotein IIb/IIla complex and initiates aggregation. Proc. Natl. Acad. Sci. USA. 82:7424-7428.

10. De Marco, L., M. Mazzuccato, M. G. Del Ben, U. Budde, A. B. Federici, A. Girolami, and Z. M. Ruggeri. 1987. Type IIB von Willebrand factor with normal sialic acid content induces platelet aggregation in the absence of ristocetin: role of platelet activation, fibrinogen, and two distinct membrane receptors. J. Clin. Invest. 80:475-482.

11. Ruggeri, Z. M., R. Lombardi, L. Gatti, R. Bader, C. Valsecchi, and T. S. Zimmerman. 1982. Type IIB von Willebrand's disease: differential clearance of endogenous versus transfused large multimer von Willebrand factor. Blood. 60:1453-1456.

12. Bellinger, D. A., T. C. Nichols, M. S. Read, R. L. Reddick, M. A. Lamb, K. M. Brinkhous, B. L. Evatt, and T. R. Griggs. 1987. Prevention of occlusive coronary artery thrombosis by a murine monoclonal antibody to porcine von Willebrand factor. Proc. Natl. Acad. Sci. USA. 84:8100-8104.

13. Weiss, H. J., J. Hawiger, Z. M. Ruggeri, V. T. Turitto, P. Thiagarajan, and T. Hoffman. 1989. Fibrinogen-independent platelet adhesion and thrombus formation on subendothelium mediated by glycoprotein IIb-IIIa complex at high shear rate. J. Clin. Invest. 83:288-297.

14. Federici, A. B., R. Bader, S. Pagani, M. L. Colibretti, L. De Marco, and P. M. Mannucci. 1989. Binding of von Willebrand factor (vWF) to glycoproteins (GP) Ib and IIb-IIIa complex: affinity is related to multimeric size. Br. J. Haematol. 73:93-99.

15. Zimmerman, T. S., J. A. Dent, Z. M. Ruggeri, and L. H. Nannini. 1986. Subunit composition of plasma von Willebrand factor: cleavage is present in normal individuals, increased in IIA and IIB von Willebrand disease, but minimal in variants with aberrant structure of individual oligomers (types IIC, IID and IIE). J. Clin. Invest. 77:947951.

16. Towbin, H., T. Stahelin, and J. Gordon. 1979. Electrophoretic transfer of proteins from polyacrylamide gels to nitrocellulose sheets: procedure and some applications. Proc. Natl. Acad. Sci. USA. 76:4350-4354.

17. Lowry, O. H., N. J. Rosebrough, A. C. Farr, and R. J. Randall. 1951. Protein measurement with Folin phenol reagent. J. Biol. Chem. 193:265-269.

18. Warren, L. 1959. The thiobarbituric acid assay of sialic acids. $J$. Biol. Chem. 234:1971-1975.

19. Fraker, D. J., and J. C. Speck. 1978. Protein and cell membrane iodinations with a sparingly soluble chloramide, 1,3,4,6-tetrachloro3a,6a-diphenylglycoluril. Biochem. Biophys. Res. Commun. 80:849857.

20. Handa, M., K. Titani, L. Z. Holland, J. R. Roberts, and Z. M. Ruggeri. 1986. The von Willebrand factor-binding domain of platelet membrane glycoprotein Ib: characterization by monoclonal antibodies 
and partial amino acid sequence analysis of proteolytic fragments. $J$. Biol. Chem. 261:12579-12585.

21. Lombardo, V. T., E. Hodson, J. R. Roberts, T. J. Kunicki, T. S. Zimmerman, and Z. M. Ruggeri. 1985. Independent modulation of von Willebrand factor and fibrinogen binding to the platelet membrane glycoprotein IIb/IIIa complex as demonstrated by monoclonal antibody. J. Clin. Invest. 76:1950-1958.

22. Ey, P. L., S. J. Prowse, and C. R. Jenkin. 1978. Isolation of pure IgG1, IgG2a and IgG2b immunoglobulins from mouse serum using protein A-Sepharose. Immunochemistry. 15:429-436.

23. Nisonoff, A., and D. J. Dixon. 1964. Evidence for linkage of univalent fragments or half-molecule of rabbit gamma globulin by the same disulfide bond. Biochemistry. 3:1338-1342.

24. De Marco, L., A. Girolami, T. S. Zimmerman, and Z. M. Ruggeri. 1986. von Willebrand factor interaction with the glycoprotein IIb/IIIa complex: its role in platelet function as demonstrated in patients with congenital afibrinogenemia. J. Clin. Invest. 77:1272-1277.

25. Walsh, P. N., D. C. B. Mills, and J. G. White. 1977. Metabolism and function of human platelets washed by albumin density gradient separation. Br. J. Haematol. 36:281-298.

26. De Marco, L., A. Girolami, S. Russell, and Z. M. Ruggeri. 1985. Interaction of asialo von Willebrand factor with glycoprotein Ib induces fibrinogen binding to the glycoprotein IIb/HIla complex and mediates platelet aggregation. J. Clin. Invest. 75:1198-1203.
27. Munson, P. J., and D. Rodbard. 1980. LIGAND: a versatile computerized approach for characterization of ligand binding system. Anal. Biochem. 107:220-239.

28. Niiya, K., E. Hodson, R. Bader, V. Byers-Ward, J. A. Koziol, E. F. Plow, and Z. M. Ruggeri. 1987. Increased surface expression of the membrane glycoprotein IIb/IIIa complex induced by platelet activation: relationship to the binding of fibrinogen and platelet aggregation. Blood. 70:475-483.

29. Ruggeri, Z. M., and T. S. Zimmerman. 1981. The complex multimeric composition of factor VIII/von Willebrand factor. Blood. 57:1140-1143.

30. Dower, S. K., C. De Lisi, J. A. Titus, and D. M. Segal. 1981. Mechanism of binding of multivalent immune complexes to Fc receptors. I. Equilibrium binding. Biochemistry. 20:6326-6334.

31. Kao, K. J., S. V. Pizzo, and P. A. McKee. 1979. Demonstration and characterization of specific binding sites for factor VIII/von Willebrand factor on human platelets. J. Clin. Invest. 63:656-664.

32. Peterson, D. M., N. A. Statopoulos, T. D. Giorgio, J. D. Hellums, and J. L. Moake. 1987. Shear-induced platelet aggregation requires von Willebrand factor and platelet membrane glycoproteins $\mathrm{Ib}$ and IIb-IIIa. Blood. 69:625-628.

33. De Marco, L., and S. S. Shapiro. 1981. Properties of human asialo-factor VIII: a ristocetin-independent platelet-aggregating agent. J. Clin. Invest. 68:321-328. 